\title{
Catheter Ablation of Atrial Flutter Using Radiofrequency Energy
}

\author{
Hugh Calkins, MD; Angel R. Leon, MD, A. Gregory Deam, MD, Steven J. Kalbfleisch, MD, \\ Jonathan J. Langberg, MD, and Fred Morady, MD
}

\begin{abstract}
Sixteen patients with type I atrial flutter underwont an attempt at radiofrequency catheter ablation (8 women, 8 men, moan age $53 \pm 11$ years). The primary criterion used to identify sites for radiofrequency energy delivery was the identification of a fractionated electrogram. Radiofrequency energy was delivered for 20 to 30 seconds. Ra diofrequency catheter ablation was acutely successful in 13 patients and unsuccessful in 3. Duiring a mean follow-up of $10 \pm 4$ months, 9 of 13 patients with a successful acute result (69\%) remained free of recurrent atrial flutter or atrial fibrillation. The ability to induce nonclinical types of atrial flutter was associated with an unsuccessful outcome. A greater proportion of electrograms reconded at successful sites demonstrated electrogram stability cormpared with unsuccessful ablation sites. None of the electrograms recorded at successful ablation sites were fractionated or had a double potential. This study demonstrates that radiofrequency catheter ablation of type I atrial flutter can be achieved safely.
\end{abstract}

(Am J Cardiol 1994;73:353-356)

From the Division of Cardiology, University of Michigan Medical Center, Ann Arbor, Michigan; Johns Hopkins Hospital, Baltimore, Maryland; and Crawford Long Hospital, Atlanta, Georgia. Manuscript reccived July 6, 1993; revised manuscript received August 11, 1993, and accepted August 16.

Address for reprints: Hugh Calkins, MD, Johns Hopkins Hospital, 600 North Wolfe Street, Camegie 530, Baltimore, Maryland 212876568.
7 he present study reports the results of radiofrequency catheter ablation of type I atrial flutter using an abbreviated mapping strategy. Mapping was performed in the region of the inferior right atrium bordered by the inferior vena cava, the tricuspid annulus and coronary sinus os. Target sites were selected based on the appearance and timing of the local electrogram. The aim of this study was to determine if this abbreviated mapping strategy is effective, to characterize the predictors of a successful outcome, and to identify the electrographic characteristics that are helpful in identifying successful target sites.

\section{METHODS}

Patient characteristics: The subjects of this study were 16 patients with type I atrial flutter (Table I) ${ }^{1,2}$ Each patient had failed or had been intolerant to treatment with a mean of $3.3 \pm 1.3$ antiarrhythmic agents including amiodarone in 4 patients (numbers 2, 6, 12, 16). The symptoms of atrial flutter included palpitations in 11 patients and dyspnea in 4 patients. Each of the patients with chronic atrial flutter had been cardioverted $\geq 2$ times.

Electrophysiologic testing: Electrophysiology tests were performed after discontinuation of antiarrhythmic agents. All patients gave written informed consent. Two standard 6Fr quadripolar electrode catheters (USCI) were positioned in the high right atrium and at His bundle position. A 7Fr bipolar electrode catheter with a 4 $\mathrm{mm}$ distal electrode and $2 \mathrm{~mm}$ interelectrode spacing was used for mapping and ablation. Surface and intracardiac electrograms were recorded at paper speeds of 25 or $100 \mathrm{~mm} / \mathrm{s}$. Bipolar electrograms were amplitied at a gain setting of $2 \mathrm{~cm} / \mathrm{mV}$, filtered at 50 to $500 \mathrm{~Hz}$, and recorded at paper speeds of $100 \mathrm{~mm} / \mathrm{s}$. A 12-lead electrocardiogram was recorded during atrial flutter and during sinus rhythm. If the patient was in atrial flutter at the start of the electrophysiology test, atrial pacing was performed at progressively shorter cycle lengths between 300 and $\mathbf{1 5 0} \mathrm{ms}$ to terminate atrial flutter. After restoration of sinus rhythm, 1 and then 2 atrial extrastimuli were delivered following an 8-beat drive train at cycle lengths of 500 and $400 \mathrm{~ms}$. If atrial flutter was not induced, atrial pacing was performed at progressively shorter cycle lengths to $150 \mathrm{~ms}$.

Mapping and ablation of atrial fiutter: In each patient, mapping efforts were concentrated in the region of the inferior right atrium bordered by the inferior vena cava, tricuspid annulus and coronary sinus os. The first criterion used to identify a site for delivery of radiofrequency energy was a fractionated atrial electrogram with a duration $>80 \mathrm{~ms}$ (Figure 1). This criterion was used based on a previous study of catheter ablation of 
atrial flutter using direct-current energy. ${ }^{3}$ If fractionated electrograms were not identified or if delivery of radiofrequency energy at sites demonstrating fractionation was unsuccessful, radiofrequency energy was delivered at sites with more discrete electrograms and early activation (Figure 2).

Once an appropriate target site was identified, radiofrequency energy was delivered between the distal electrode of the ablation catheter and a large skin electrode
(Figure 3). Twenty-five to $35 \mathrm{~W}$ were delivered during atrial flutter for 20 to 30 seconds. If atrial flutter terminated, a second application was delivered. Thirty minutes later, the inducibility of atrial flutter was reevaluated. In each patient, the time required for the ablation procedure was recorded.

After the procedure, patients underwent electrocardiographic monitoring for 1 or 2 days. Creatine kinase and MB isoenzyme concentrations were measured every

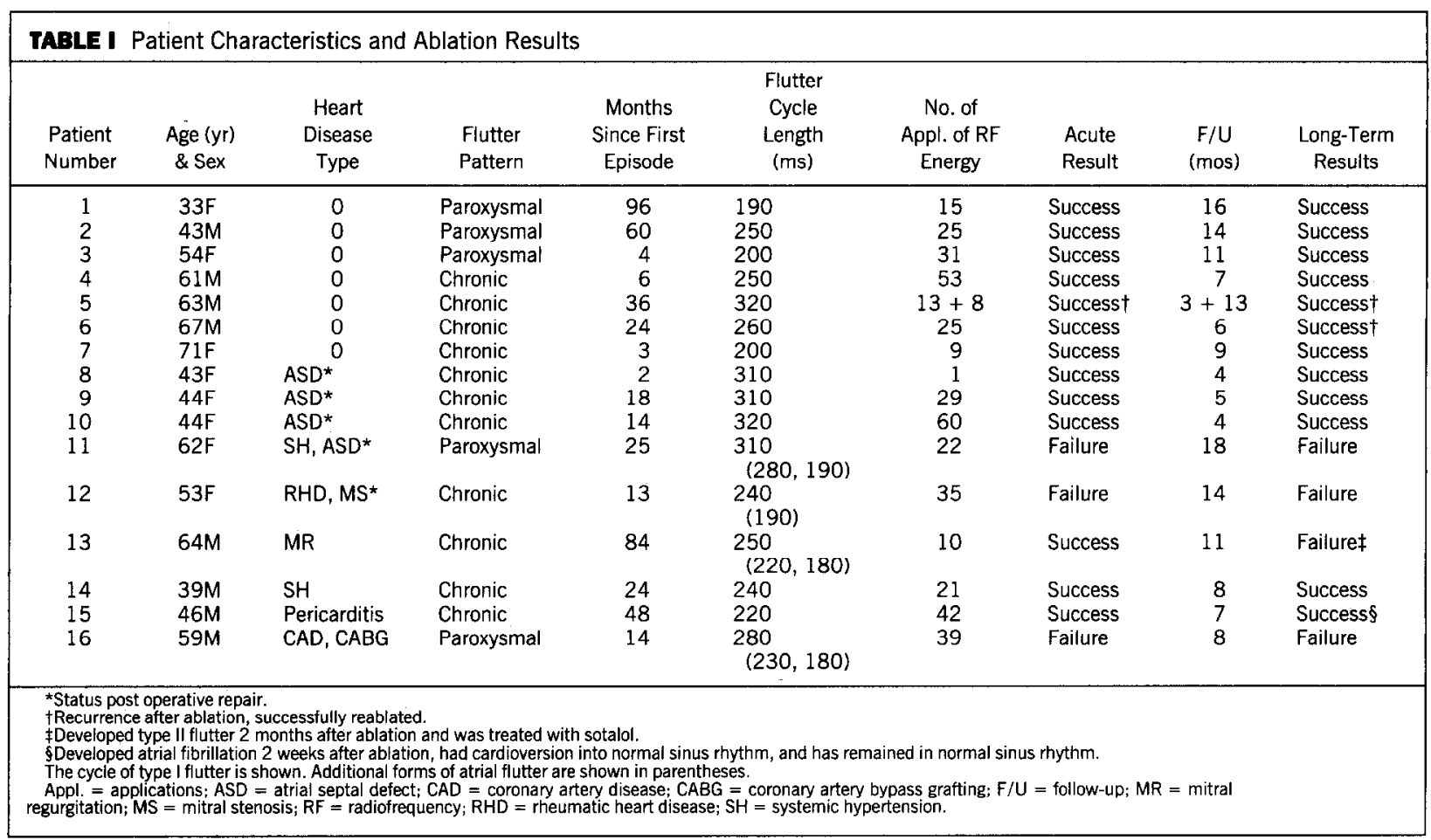

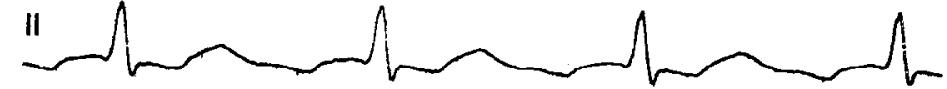

CS os
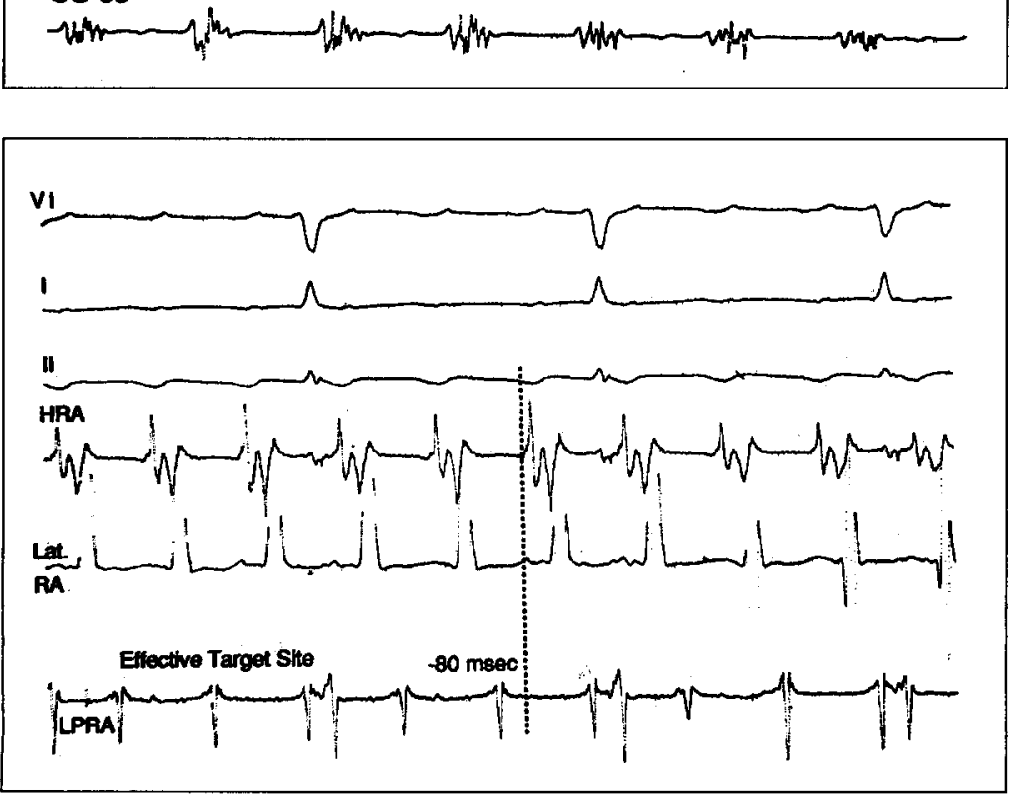

FICURE 1. Recording obtained at an ablation site at which a prolonged fragmented electrogram is observed. Surface electrocardiographic lead II and the electrogram recorded with the ablation catheter near the coronary sinus (CS) os are shown.

FGURE 2. Recordingts obtained during atrial flutter at a successful ablation site. Surface leads $V_{1}, I$ and II and the intracardiac eloctrograms recorded in the high right atrium (HRA), lateral (Lat.) right atrium (RA), and at the ablation site in the low posterior right atrium (LPRA) are shown. The narrow atrial electrogram recorded at the successful ablation site begins $80 \mathrm{~ms}$ before onset of the fiutter wave. A ventricular component is also observed with an atrioventricular ratio of approximately 1. 
8 hours for 24 hours. An echocardiogram was obtained after the procedure in 12 of the 16 patients.

Follow-up: Patients with a successful acute result, defined as the absence of inducible atrial flutter at the completion of the ablation session, were discharged without antiarrhythmic therapy. Each patient was seen every 2 to 6 months for follow-up. A Holter monitor was obtained 2 months after the ablation procedure. If symptoms recurred, a 12-lead electrocardiogram was obtained. If no arrhythmia was present, the patient was provided with an event monitor. Each patient was also interviewed by telephone to obtain follow-up information.

Analysis of electrograms: The characteristics of local electrograms recorded at successful and unsuccessful sites of radiofrequency catheter ablation were determined in each patient in whom the outcome was successful. If $>10$ applications of radiofrequency energy were required, the electrogram characteristics of the successful and the first 10 unsuccessful sites were determined. Each electrogram was analyzed in a blinded fashion.

The amplitude, timing and morphology of the atrial component of each local electrogram was determined. Morphologic features evaluated included the stability of the local electrogram, the presence of fractionation or the presence of a double potential. Electrograms were classified as unstable if there was $>25 \%$ change in the amplitude of the atrial component of the local electrogram or appearance or disappearance of a major deflection in the local electrogram. The onset and activation time of the atrial component of the local electrogram were also determined relative to the onset of the flutter wave in lead II. The electrogram onset was defined as the first deflection from baseline with a slope $>45$ degrees at a paper speed of $100 \mathrm{~mm} / \mathrm{s}^{4}$. The activation time was defined as the point of maximal amplitude of the local atrial electrogram. 4,5

Statistical analysis: Continuous variables are expressed as mean $\pm 1 \mathrm{SD}$. Determination of predictors of success based on clinical variables or electrographic characteristics was performed using Student's $t$ test or by contingency table analysis. A p value $<0.05$ was considered significant.
TABLE II Comparison of Successful and Failed Sites of

Radiofrequency Energy Delivery

\begin{tabular}{|c|c|c|c|}
\hline Variable & $\begin{array}{l}\text { Successful } \\
\text { Sites } \\
(n=13)\end{array}$ & $\begin{array}{l}\text { Failed Sites } \\
(n=119)\end{array}$ & $p$ Value \\
\hline Onset (ms) & $-62 \pm 38$ & $-72 \pm 55$ & 0.6 \\
\hline Activation (ms) & $-45 \pm 29$ & $-60 \pm 52$ & 0.6 \\
\hline Duration (ms) & $58 \pm 19$ & $86 \pm 44$ & 0.08 \\
\hline Duration $\geq 80 \mathrm{~ms}$ & $1(8 \%)$ & $44(37 \%)$ & 0.19 \\
\hline Amplitude (mV) & $0.9 \perp 0.4$ & $0.8 \pm 0.8$ & 0.42 \\
\hline Stability & $13(100 \%)$ & $82(69 \%)$ & 0.02 \\
\hline \multicolumn{4}{|l|}{ Morphology } \\
\hline Fractionated & $0(0 \%)$ & $77(65 \%)$ & $<0.001$ \\
\hline Double potential & $0(0 \%)$ & $36(30 \%)$ & $<0.02$ \\
\hline $\begin{array}{l}\text { Ventricular } \\
\text { component }\end{array}$ & $4(31 \%)$ & $42(35 \%)$ & 0.9 \\
\hline
\end{tabular}

\section{RESULTS}

Basoline characteristics of atrial fiutter: Thirteen patients were in atrial flutter at the start of the electrophysiology test and 3 were in sinus rhythm. Type I atrial flutter was reproducibly initiated and tennirated with atrial pacing in 15 patients. In the remaining patient, atrial flutter was incessant. The mean atrial flutter cycle length was $258 \pm 44 \mathrm{~ms}$ (range 190 to 320 ). In 4 patients, other types of atrial flutter, which did not have inverted flutter waves in the inferior leads, were induced (mean cycle length $220 \pm 40 \mathrm{~ms}$; range 180 to $230 \mathrm{~ms}$ ).

Catheter ablation results: Radiofrequency catheter ablation was acutely successful in 13 patients $(81 \%)$ and unsuccessful in 3 (Table I). The mean number of radiofrequency applications in patients with a successful result was $26 \pm 17$, compared with $32 \pm 9$ in those with an unsuccessful result. In each patient the successful ablation site was located in the region of the coronary sinus os. Two of the 3 patients in whom catheter ablation was not successful underwent atrioventricular junction ablation and insertion of a permanent pacemaker, and the third patient was treated with amiodarone.

During a mean follow-up of $10 \pm 4$ months, 9 of the 13 patients $(69 \%)$ with a successful acute result, representing $56 \%$ of the patient population, remained free of recurrent atrial flutter or atrial fibrillation. Two patients (numbers 5 and 6) experienced recurrent type I atrial

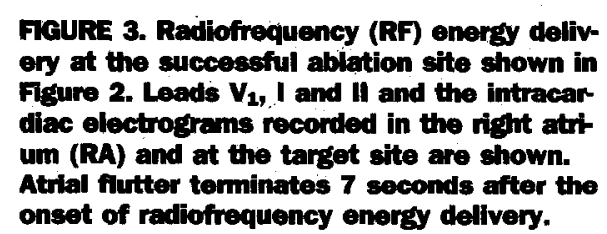

FGURE 3. Radiofrequency (RF) energy delivFinge 2 teat $V$, and diac electrograms reconded in the right atrium (RA) and at the tardet site are shown. onset of radiofrequency energy delivery.

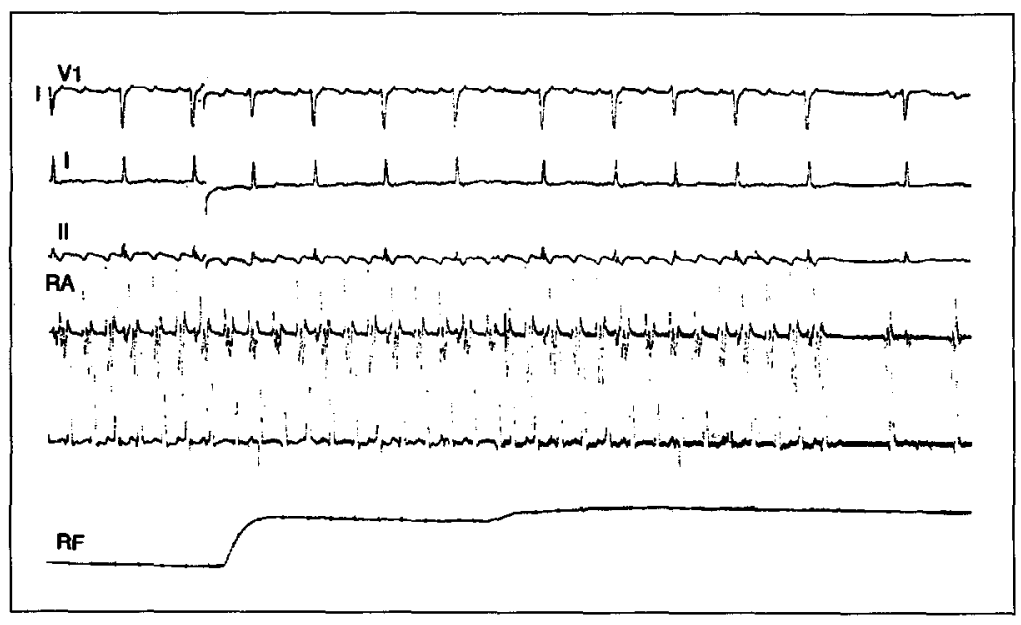


flutter 3 and 6 weeks after the initial ablation session and underwent a successful second attempt. The successful site was similar to that identified during the first attempt. One patient (number 15) developed atrial fibrillation 2 weeks after the ablation session and achieved cardioversion to sinus rhythm, and 1 patient (number 13) developed type II atrial flutter 2 months after the initial session and was treated with sotalol. Thus, 9 of the 13 patients $(62 \%)$ with a successful acute result have remained in sinus rhythm without antiarrhythmic drug therapy.

Predictors of success: The inability to induce nonclinical types of atrial flutter was associated with a successful outcome $(p=0.01)$. The success rate was $100 \%$ in patients who had only type I atrial flutter, compared with $25 \%$ in patients who also had other types of inducible atrial flutter. Variables that were not associated with outcome included patient age, the duration of atrial flutter, a history of prior cardiac surgery, and the cycle length of the atrial flutter.

Comparison of electrograms at successful and unsucceseful tarfet sites: The characteristics of the electrograms recorded at successful and unsuccessful sites of radiofrequency energy delivery are described in Table II. A greater proportion of electrograms recorded at successful sites demonstrated electrographic stability $(p=0.02)$. None of the electrograms recorded at successful ablation sites were fractionated or had a double potential. Each of the other electrographic parameters that was evaluated did not differ at successful and unsuccessful ablation sites.

Duration and complications of ablation procedures: Mean duration of the ablation procedure was $114 \pm 49$ minutes. No patient developed a complication. Mean peak creatine kinase concentration was $105 \pm 112 \mathrm{IU} /$ liter (normal 30 to 240). No patient demonstrated an elevation in the creatine kinase-MB fraction or a pericardial effusion after the ablation procedure.

\section{DISCussion}

Main findings: Results of this study demonstrate that radiofrequency catheter ablation of type I atrial flutter using an abbreviated mapping protocol can be performed safely. Although ablation of atrial flutter is accomplished in most patients during the initial ablation session, recurrence of atrial fibrillation or atrial flutter are not uncommon. The success rate may depend on the underlying substrate of the flutter, and in this series the outcome was uniformly successful in patients with only a single morphologic feature of atrial flutter. This finding suggests that the substrate of the reentrant circuit in patients with $>1$ morphologic feature of atrial flutter differs from the substrate of idiopathic type I atrial flutter and may be less amenable to radiofrequency ablation perhaps because of prior right atrial scarring. ${ }^{6}$ The absence of fractionated electrograms at successful sites suggests that fractionated electrograms are not a marker of a critical discrete component of the flutter circuit.
Eectrographic characteristics at succeseful abla tion sites: The following characteristics defined electrograms at successful target sites: (1) early onset and activation relative to the flutter wave, (2) a duration $<80$ ms, (3) electrographic stability, and (4) absence of double potentials or fragmentation. However, the only electrographic characteristic that differed between successful and unsuccessful sites was electrographic stability. This may reflect the importance of electrode stability. These results suggest that fractionated elcetrograms or those with double potentials may not be markers of a critical discrete component of the reentrant circuit that can be ablated with radiofrequency energy.

Comparison with prior studies: The $81 \%$ acute efficacy of radiofrequency catheter ablation of type I atrial flutter in this series is somewhat lower than the $100 \%$ success rate reported by Feld et al, ${ }^{2}$ but is similar to the $78 \%$ success rate reported by Cosio et al. ${ }^{7}$ The lower efficacy of catheter ablation in this series compared with Feld et $\mathrm{al}^{2}$ may reflect differences in mapping technique. In this study, mapping was localized to the inferior right atrium, and entrainment mapping was not used. In contrast, in the previous study, the activation sequence of the entire right atrium was mapped, and target sites for radiofrequency catheter ablation were selected on the basis of electrographic timing and observation of concealed entrainment. The lower efficacy of catheter ablation in this study may also reflect differences in the patient population. If the patients in this study who had multiple morphologic characteristics of atrial flutter are excluded, the acute success rate would be $100 \%$, similar to that reported by Feld et al. ${ }^{2}$

Fifty-six percent of patients in whom catheter ablation was attempted remained in sinus rhythm without all antiarrhythmic agents during a mean follow-up of $10 \pm$ 4 months. These results are similar to the $58 \%$ long-term success rate achieved by Feld et $\mathrm{al}^{2}$ and the $44 \%$ longterm success rate achieved by Cosio et al. ${ }^{7}$ However, it must be recognized that the duration of follow-up in each of these studies remains $<1$ year, and longer follow-up is required to determine more precisely the true "long-term" efficacy of the procedure.

1. Wells JL, MacLean WAH, James TN, Waldo AL. Characterization of atrial flutter: studies in man after open heart surgery using fixed atrial electrodes. Circulation 1979;60:665-673.

2. Feld G, Fleck RP, Chen PS, Boyce K, Bahnson TD, Stein JB, Calisi CM, Ibarra M. Radiofrequency catheter ablation for the treatment of human type I atrial flutter. Identification of a critical zone in the reentrant circuit by endocardial mapping techniques. Circulation 1992;86:1233-1240.

3. Saoudi N, Attallah G, Kirkorian G, Touboul P. Catheter ablation of the atrial myocardium in human type I atrial flutter. Circulation 1990;81:762-771.

4. Gallagher JJ, Kasel JH, Cox JL, Smith WM, Ideker RE, Smith WM. Techniques of intraoperative electrophysiologic mapping. Am J Cardiol 1982;49:221-240. 5. Paul T, Moak JP, Morris C, Garson A. Epicardial mapping: how to measure local activation? PACE 1990;13:285-292.

6. Boyden PA, Frame LH, Hoffman BF. Activation mapping of reentry around an anatomic barrier in the canine atrium. Circulation 1989;79:406-416.

7. Cosio FG, Lopez-Gil M, Goicolea A, Arribas F, Barroso JL. Radiofrequency ablation of the inferior vena cava-tricuspid valve isthmus in common atrial flutter. Am J Cardiol 1993;71:705-709. 